\title{
Avances del proceso de formalización de derechos de comunidades nativas en la Amazonía peruana (2014-2018)
}

\author{
Iliana Monterroso y Anne M. Larson
}

\section{Mensajes Clave}

- La movilización de organizaciones indígenas y de sociedad civil han sido claves para incorporar el tema del reconocimiento del derecho colectivo en la agenda política, reducir la brecha en la formalización de comunidades nativas y promover avances en la implementación.

- Los cambios mas recientes en las regulaciones sobre la formalización se han dado a través de la aprobación de lineamientos y reglamentos específicos; buscan estandarizar y esclarecer criterios técnicos sobre procesos específicos y agilizar procedimientos.

- Las oportunidades actuales de financiamiento incluyen iniciativas relacionadas con REDD+y las negociaciones sobre el clima que han incorporado en sus metas la titulación de las comunidades nativas, pero no está claro que llegarán al importante paso del registro de los títulos.

- Situaciones de conflicto, como Bagua y Saweto, han promovido la opinión pública en favor de los derechos colectivos de pueblos indígenas.

\section{Introducción}

Desde 1974, el Gobierno peruano ha formalizado los derechos de propiedad colectiva al titular más de 1300 comunidades nativas sobre más de 12 millones de hectáreas (IBC, 2016). Este reconocimiento es importante para estos pueblos que habitan la Amazonía peruana y dependen de manera directa de estos bosques, y también tiene implicaciones en las condiciones de los bosques que estos ocupan y que representan el 17\% del área de bosques a nivel nacional (MINAM 2016) ' . A pesar estos avances, existe una brecha considerable en lo que respecta al proceso de formalización de las tierras reclamadas por los pueblos indígenas. Según el SICNA-IBC (2016), más de 600 comunidades nativas (unos 5,5 millones de hectáreas) quedan aún por titular. La ausencia de un registro nacional dificulta determinar la extensión y el número de reclamos pendientes (Defensoría del Pueblo 2014). Por ejemplo, según la Asociación Interétnica para el Desarrollo de la Selva Peruana (AIDESEP 2016), existen reclamos sobre 20 millones de hectáreas de tierras y bosques. Estos incluyen reivindicaciones

1 Aunque este brief no trata el tema, vale resaltar que la relación entre la tenencia colectiva de pueblos indígenas y la condición de los bosques dentro de las comunidades tituladas ha sido analizada en la literatura especializada (e.g. Blackman et al. 2017; Schleicher et al. 2017). por el reconocimiento y la titulación de comunidades nativas, ampliación de comunidades nativas ya tituladas y otros mecanismos que permiten el reconocimiento de derechos de gestión a grupos indígenas sobre áreas protegidas, como las reservas comunales ${ }^{2}$. También se incluyen las demandas por el establecimiento de nuevas reservas territoriales para pueblos aislados y en situación de contacto inicial (AIDESEP 2013).

Según el marco regulatorio vigente (Ley de Comunidades Nativas y de Desarrollo Agrario de la Selva y Ceja de Selva, Decreto Ley 22175 de 1978), las comunidades nativas formalizan sus derechos a la tierra y el bosque a partir de un proceso que tiene dos resultados diferentes: a) la demarcación y titulación de tierras de uso agrario y b) la firma de un contrato de cesión en uso sobre tierras de uso forestal. Ambos requieren que las comunidades nativas sean antes reconocidas legalmente (Resolución Ministerial 0435-2016-MINAGRI). Esta diferencia en el derecho otorgado sobre áreas de bosque se da desde 1975, cuando se estableció que el patrimonio forestal es de dominio público; desde entonces,

2 Las reservas comunales son una categoría de área protegida que reconoce derechos de gestión a las comunidades (Régimen Especial de Administración de Reservas Comunales, Resolución de Intendencia 019-2005-INRENA-IANP). 
las tierras con aptitud forestal y de protección no pueden titularse. Hasta 2017, se utilizaba un estudio de suelos (RM 0355-2015-MINAGRI) para definir el uso mayor y establecer qué área otorgar bajo título agrario (área de uso agrícola y pecuario) y qué área entregar bajo contrato de cesión en uso (área de uso forestal). Actualmente, se utiliza una evaluación agrológica que elimina el análisis químico, pero mantiene el uso de calicatas, como técnica para realizar la clasificación de la capacidad de uso sobre el área reclamada (RM 0194-2017-MINAGRI, véase Tabla 1).

A pesar de que el proceso de formalización de comunidades nativas se inició hace más de 40 años, los cambios en el marco regulatorio han afectado el avance en la implementación de las normas. Aún hay comunidades nativas pendientes de reconocimiento y titulación, así como comunidades nativas que aún no cuentan con contratos de cesión en uso que formalicen sus derechos sobre los bosques en las tierras categorizadas bajo este uso. La implementación de estas normas continúa en un contexto complejo, con intereses diversos marcados por cambios en las prioridades de los Gobiernos de turno, así como por la movilización de organizaciones indígenas y de sociedad civil que han sido claves para reducir la brecha en el reconocimiento del derecho colectivo.

Esta publicación es parte del "Estudio comparativo global sobre diseño e implementación de reformas de tenencia en tierras forestales" de CIFOR ${ }^{3}$, y tiene como objetivo reflexionar sobre el proceso de reconocimiento de derechos territoriales de comunidades nativas en la Amazonía peruana a partir de los cambios recientes en el proceso de formalización. Este documento analiza los avances más importantes en materia de legislación, institucionalidad y financiamiento relacionados con la formalización de derechos a la tierra y el bosque de comunidades nativas. Este análisis es producto de la síntesis de una mesa de discusión con actores clave realizada en noviembre de $2017^{4}$ y de otras discusiones ${ }^{5}$ que abordan el tema de la titulación y del análisis de los procedimientos de formalización de derechos de comunidades nativas, en particular las diferencias entre el marco regulatorio y su implementación (CIFOR y WRI 2017).

3 Véase http://www.cifor.org/gcs-tenure/

4 La mesa de discusión "Tenencia colectiva, interculturalidad y género en la formalización de comunidades nativas" se llevó a cabo en lquitos, Loreto, con los objetivos de (1) discutir los actuales desafíos y obstáculos a la implementación de procesos de formalización del derecho colectivo, a partir de experiencias concretas de reconocimiento y titulación de comunidades nativas; y (2) revisar qué experiencias de titulación existentes, promovidas por los principales agentes gubernamentales, de cooperación internacional y ONG, incorporan los componentes de género (de manera directa o indirecta) e interculturalidad, y revisar su alcance y replicabilidad. Los resultados de esto se presentan en Durán et al. (2018).

5 Nos referimos por ejemplo al grupo de trabajo sobre titulación (conformado en el marco de la Mesa Nacional sobre Reducción de Emisiones de gases de efecto invernadero causadas por la Deforestación y Degradación de los bosques, REDD+), constituido principalmente por ONG que trabajan en dicho tema.

\section{Cambios en la legislación, la institucionalidad y el financiamiento}

Recientemente, un observador comentaba que, en Perú, más que en otros países de la región latinoamericana, hay una tendencia a separar sectores y metas: en particular, es difícil mantener una visión común entre titulación de tierras y manejo sostenible de bosques. Existen desencuentros no solamente entre sectores sino también entre los niveles nacional y subnacional, donde cada sector dice que los vacíos son responsabilidad del otro (Defensoría del Pueblo 2014). El marco legal también es extraordinariamente complejo: un compendio de leyes y normas sobre la formalización suma más de 700 páginas, y ni siquiera los abogados están de acuerdo sobre la interpretación de algunos procedimientos establecidos en la norma.

Los trágicos eventos de Bagua en $2009^{6}$ y la muerte de cuatro líderes asháninkas de la comunidad de Saweto en 2014 generaron un renovado interés por los derechos indígenas en el escenario político del país (Monterroso et al. 2017). Posteriormente a estos eventos, la movilización de organizaciones indígenas y otros defensores de derechos indígenas se centró en promover cambios en el marco institucional y legal para mejorar la implementación y acelerar el proceso de reconocimiento y formalización de los derechos de comunidades nativas. Como resultado de estas movilizaciones, se ha logrado que compromisos internacionales y nacionales que buscan enfrentar las causas de la deforestación en el Perú incluyan, dentro de sus objetivos, metas relacionadas con la regularización de comunidades nativas y generen nuevas oportunidades de financiamiento (Bolaños 2017). Este documento analiza los principales avances a partir de los cambios en la legislación, la institucionalidad y el financiamiento, que se derivan de estos sucesos.

\section{Cambios en la legislación}

Después de Bagua, ha habido dos reformas importantes en la legislación que afectan a comunidades nativas (Monterroso et al. 2017)7. En 2011 se aprobó la Ley del Derecho a la Consulta Previa a los Pueblos Indígenas u Originarios (Ley 29785), que buscaba establecer el marco para el reconocimiento del derecho de los pueblos indígenas al consentimiento libre, previo e informado 8 . La segunda fue la aprobación de la Ley Forestal y de Fauna Silvestre (Ley 29763) en 2011, que además se convirtió en el primer proceso de consulta para una legislación en aplicación de la Ley 29785. El proceso de consulta de sus cuatro reglamentos se extendió hasta el año 2015.

6 En 2009, una confrontación entre pueblos indígenas y autoridades policiales durante una protesta contra cambios propuestos a las regulaciones forestales en Bagua terminó con la muerte de 33 personas 7 Es importante resaltar otros cambios recientes en la legislación como las Leyes 30230 (2014) y 30327 (2015) que incluyen disposiciones específicas que también pueden afectar a comunidades nativas (IBC, 2016; Red Muqui y GRUFIDES 2015).

8 Sin embargo, es importante aclarar que consentimiento y consulta no son lo mismo. 
Tabla 1. Principales cambios en las regulaciones relacionadas con la formalización de comunidades nativas en 2014-2017

\begin{tabular}{lll}
\hline Año & Regulación & Descripción \\
\hline 2014 & Resolución Ministerial 0547-2014-MINAGRI & Resolución aprobada a partir de la movilización política para resolver \\
& el proceso de titulación de la comunidad de Saweto, que se encontra- \\
& ba suspendido debido a su superposición con bosques de producción \\
& permanente (BPP). \\
& Garantiza la demarcación y titulación ininterrumpidas de una comu- \\
& nidad nativa a pesar de la superposición con áreas clasificadas como \\
& bosque de producción permanente. \\
& Plantea que el proceso de demarcación y titulación de una comunidad \\
& nativa no puede ser suspendido por su superposición con áreas de \\
& BPP (artículo 1). \\
& Define los pasos para modificar el tamaño de los bosques de produc- \\
& ción con el fin de facilitar la demarcación y titulación de las comuni- \\
& dades nativas en los casos en que existe superposición. \\
& Determina que los Gobiernos Regionales son responsables de infor- \\
& mar a SERFOR (Servicio Nacional Forestal y de Fauna Silvestre) para \\
& poder realizar el proceso de redimensionamiento del BPP.
\end{tabular}

2015 Decreto Supremo 021-2015. Reglamento para la Gestión Forestal y de Fauna Silvestre en Comunidades Nativas y Comunidades Campesinas
- Aprueba disposiciones específicas para la gestión de bosques en tierras de comunidades nativas y campesinas.

- Crea nuevas estructuras a nivel de los Gobiernos Comunales y Locales para promover su participación y la participación de comunidades en la organización de actividades de gestión forestal a nivel local.

2016 Resolución Ministerial 0435-2016-MINAGRI y Resolución Ministerial 0589-2016-MINAGRI. Procedimiento de Reconocimiento e Inscripción Administrativa de la Personería Jurídica de Comunidades Nativas

2017 Resolución Ministerial 0194-2017-MINAGRI. Lineamientos para la ejecución del proceso de evaluación agrológica de las tierras de las comunidades nativas y la clasificación por su capacidad de uso mayor a nivel de grupo con fines de titulación específicos para estandarizar los procedimientos para el reconojurídicas.

- Sustituye el uso de análisis químico de suelo para categorizar el mejor uso de la tierra como un prerrequisito para la titulación de las una evaluación agrológica algo simplificada para la clasificación de su capacidad de uso.
- Establece lineamientos para la ejecución del proceso de reconocimiento de comunidades nativas como sujeto jurídico.

- Proporciona a los Gobiernos Regionales directrices y criterios técnicos cimiento de nuevas comunidades nativas y su registro como entidades comunidades nativas (Resolución Ministerial 0355-2015-MINAGRI), por

- Define que la responsabilidad de llevar a cabo dicha evaluación corresponde a los Gobiernos Regionales de las regiones de selva y ceja de selva, en el marco del ejercicio de la función n) del artículo 51 de la Ley 27867, Ley Orgánica de Gobiernos Regionales.

2017 Resolución Ministerial 0370-2017-MINAGRI. Lineamientos para Georreferenciar el Plano de Demarcación Territorial de Comunidades Nativas Tituladas
- Establece, uniforma, esclarece, viabiliza y agiliza criterios técnicos y legales empleados por parte de los Gobiernos Regionales de selva y ceja de selva para realizar el proceso de georreferenciación del plano de demarcación de comunidades nativas (artículo 1).

- Define que la responsabilidad de llevar a cabo el proceso de georreferenciación corresponde a los Gobiernos Regionales.

Fuente: basado en la revisión y actualización de datos publicados por Monterroso et al. (2017), IBC (2016) y Baldovino (2016).

Posteriormente a 2014, los principales cambios en las regulaciones sobre la formalización se han dado a través de la aprobación de lineamientos y reglamentos específicos que buscan, de acuerdo a sus objetivos, estandarizar y esclarecer criterios técnicos sobre procesos específicos y agilizar procedimientos. Estos se describen en la Tabla 1.

\section{Cambios en la institucionalidad}

Además de las reformas en la regulación, en los últimos 10 años ha habido cambios importantes en la institucionalidad 
competente9. En 2009 se completó el proceso de transferencia de las competencias a los Gobiernos Regionales iniciado en 2002 (DS 064-2009-PCM). Este proceso ha sido lento y confuso y se ha caracterizado por la ausencia de recursos financieros y humanos necesarios para asegurar su implementación (Zamora y Monterroso 2017). Además, se planteaba la necesidad de establecer un ente rector que tuviera a su cargo la elaboración de lineamientos, que administrara y consolidara la información del catastro a nivel nacional, y que asegurara el cumplimiento del proceso de saneamiento físico legal y formalización de la propiedad agraria, incluidas las tierras de las comunidades nativas (IBC 2016, 38-39). No fue sino hasta 2014 que el Decreto Supremo 001-2013-AG definió al MINAGRI (Ministerio de Agricultura y Riego) como ente rector y creó la Dirección de Saneamiento de la Propiedad Agraria y Catastro Rural (DISPACR) (DS 008-2014-MINAGRI).

En 2017, se volvió a modificar el Reglamento de Organización y Funciones (ROF) del Ministerio de Agricultura y Riego a través del Decreto Supremo 001-2017-MINAGRI, que convirtió la DISPACR en la Dirección General de Saneamiento de la Propiedad Agraria y Catastro Rural (DIGESPACR) dentro del Viceministerio de Políticas Agrarias. Esta decisión representa una oportunidad para fortalecer el trabajo del ente rector, asegurar condiciones para disponer de más personal y presupuesto, brindar un mejor apoyo a los Gobiernos Regionales, articular la coordinación del trabajo que realizan los proyectos y otras iniciativas de la sociedad civil, y consolidar el sistema de Catastro Nacional como única base de datos que integre la información de las comunidades nativas en proceso de regularización.

\section{Nuevas fuentes de financiamiento}

Los cambios en la opinión pública en favor de los derechos colectivos indígenas impulsaron un debate sobre los desafíos para la implementación de las reformas existentes y permitieron que se incorporara nuevamente el tema en la agenda política (Monterroso et al. 2017). A partir de 2014, y con más fuerza en 2016, diversas iniciativas con financiamiento internacional en Perú incluyeron entre sus metas la titulación y el reconocimiento de las comunidades nativas (IBC 2016; MINAM-DCI 2018).

Si bien los resultados de estos proyectos se centran en objetivos ambientales, como la reducción de emisiones derivadas de la deforestación y la degradación de los bosques (REDD+), algunos también incluyen el reconocimiento, la demarcación y la titulación de comunidades indígenas como resultados intermedios para

9 Desde 1974, la oficina responsable de la demarcación y la titulación de los derechos colectivos a la tierra ha cambiado en varias ocasiones y ha estado dentro y fuera del ámbito del Ministerio de Agricultura y Riego. Desde 1992 hasta 2006, dicha competencia estuvo a cargo del Proyecto Especial de Titulación de Tierras (PETT), dentro del Ministerio de Agricultura y Riego. En 2006, las responsabilidades del PETT, entre ellas la titulación de comunidades nativas, fueron transferidas al Organismo de Formalización de la Propiedad Informal (COFOPRI) en el Ministerio de Vivienda, Construcción y Saneamiento. Al año siguiente, las responsabilidades del COFOPRI fueron transferidas a los Gobiernos Regionales (Decretos Supremos 088-2008-PCM y 064-2009-PCM). evitar la deforestación y la degradación (Bolaños 2017). Debido a estas iniciativas, procesos de titulación de comunidades nativas han avanzado en regiones de la Amazonía, en particular en Ucayali, San Martín y Loreto, desde 2014 ${ }^{10}$. Para el periodo 2016-2020 se han identificado al menos 10 proyectos (Tabla 2).

De acuerdo con actores regionales y locales, el proceso de regularización de las comunidades nativas termina con la inscripción del derecho formal (títulos y contratos de cesión en uso) en Registros Públicos (Zamora y Monterroso 2017). Esto es coherente con la regulación vigente (DS 003-79-AA, Resolución SUNARP 05-2013-SUNARP/SN). No obstante, las metas establecidas en los proyectos (Tabla 2) no identifican claramente si el proceso de titulación termina con la emisión y entrega del título o si se contempla su registro.

\section{Conclusión}

Este documento analiza los cambios recientes en la legislación, la institucionalidad y el financiamiento dirigidos a la formalización de comunidades nativas en Perú. La movilización de organizaciones indígenas y de sociedad civil ha sido clave para incorporar el tema del reconocimiento del derecho colectivo en la agenda política. No obstante, los avances en la legislación han sido fuertemente influidos por los resultados de situaciones de conflicto como Bagua o Saweto. Estos han promovido la opinión pública en favor de los derechos colectivos de pueblos indígenas y han sido fundamentales para promover cambios en el rumbo de los procesos políticos, así como un debate sobre los desafíos para la implementación de las reformas existentes. Las oportunidades actuales de titulación incluyen iniciativas relacionadas con REDD+y las negociaciones sobre el clima.

\section{Agradecimientos}

El "Estudio comparativo global sobre diseño e implementación de reformas de tenencia en tierras forestales" de CIFOR se desarrolla gracias al apoyo financiero de la Comisión Europea y el Fondo Mundial para el Medio Ambiente (FMAM) y cuenta con el respaldo técnico del Fondo Internacional de Desarrollo Agrícola (FIDA) y la Organización de las Naciones Unidas para la Alimentación y la Agricultura (FAO). También deseamos agradecer a Sondra Wentzel, Omaira Bolaños, Safia Aggarwal y Juan Pablo Sarmiento, por sus revisiones y comentarios. Este estudio forma parte del Programa sobre Políticas, Instituciones y Mercados (PIM), liderado por el Instituto Internacional de Investigación sobre Políticas Alimentarias (IFPRI); y del Programa de Investigación del CGIAR sobre Bosques, Árboles y Agroforestería (FTA), liderado por CIFOR. Las opiniones expresadas en este documento representan el análisis de las autoras y no necesariamente reflejan la opinión de CIFOR, CGIAR y las organizaciones donantes.

10 En menor medida, otras regiones de trabajo incluyen Madre de Dios (iniciativa del Fondo de Tenencia) y la selva central (proyecto PTRT3). Tabla 2 hace referencia a iniciativas que benefician a comunidades nativas, y no aborda la formalización del derecho colectivo a comunidades campesinas bajo otra forma de reconocimiento jurídico. 
Tabla 2. Proyectos que promueven la formalización de comunidades nativas (CC. NN.) en la Amazonía*

\begin{tabular}{|c|c|c|c|c|c|c|c|}
\hline Proyecto & $\begin{array}{l}\text { Entidad a } \\
\text { cargo }\end{array}$ & $\begin{array}{l}\text { N. }{ }^{\circ} \text { CC. } \\
\text { NN. por } \\
\text { reconocer }\end{array}$ & $\begin{array}{l}\mathrm{N} .{ }^{\circ} \text { CC. NN. por } \\
\text { titular/ ampliar }\end{array}$ & $\begin{array}{l}\text { Hectáreas } \\
\text { (aprox.) }\end{array}$ & $\begin{array}{c}\mathrm{N} .{ }^{\circ} \mathrm{CC} . \mathrm{NN} . \\
\text { georreferenciadas }\end{array}$ & $\begin{array}{l}\text { Año de } \\
\text { término del } \\
\text { proyecto }\end{array}$ & $\begin{array}{l}\text { Regiones de } \\
\text { intervención }\end{array}$ \\
\hline $\begin{array}{l}\text { 1. Programa de } \\
\text { Desarrollo Alternativo }\end{array}$ & DEVIDA & 0 & 6 & 24.336 & 0 & 2016 & Pasco \\
\hline $\begin{array}{l}\text { 2. Apoyo a la } \\
\text { implementación de } \\
\text { la DCI (Declaración } \\
\text { Conjunta de Intención) } \\
\text { entre Perú, Noruega y } \\
\text { Alemania sobre REDD+ }\end{array}$ & WWF & 0 & 35 & 150.000 & 0 & 2017 & Loreto \\
\hline $\begin{array}{l}\text { 3. Ampliación del } \\
\text { servicio de catastro, } \\
\text { titulación y registro } \\
\text { de tierras en las } \\
\text { comunidades nativas } \\
\text { de las cuencas de los } \\
\text { ríos Pastaza, Tigre, } \\
\text { Corrientes y Marañón } \\
\text { de las provincias de } \\
\text { Datem del Marañón y } \\
\text { Loreto, región Loreto }\end{array}$ & $\begin{array}{l}\text { DIGESPACR/ } \\
\text { GORE Loreto }\end{array}$ & 0 & 60 & 658.072 & 40 & 2017 & Loreto \\
\hline $\begin{array}{l}\text { 4. Programa de } \\
\text { Desarrollo Alternativo } \\
\text { Integral y Sostenible } \\
\text { (PIRDAIS) }\end{array}$ & DEVIDA & 2 & 10 & 32.758 & 0 & 2017 & Junín \\
\hline 5. Proyecto PNUD-DCI & PNUD-PNCB & 0 & 68 & 310.000 & 0 & 2018 & $\begin{array}{l}\text { San Martín, } \\
\text { Ucayali, Loreto }\end{array}$ \\
\hline $\begin{array}{l}\text { 6. Mecanismo Dedicado } \\
\text { Específico (MDE) } \\
\text { SAWETO }\end{array}$ & $\begin{array}{l}\text { AIDESEP / } \\
\text { CONAP / WWF }\end{array}$ & 310 & 130 & 780.000 & 0 & 2020 & $\begin{array}{l}\text { Loreto, Ucayali, } \\
\text { Madre de Dios, } \\
\text { Amazonas, } \\
\text { Junín, San } \\
\text { Martín, } \\
\text { Huánuco. }\end{array}$ \\
\hline $\begin{array}{l}\text { 7. Catastro, titulación } \\
\text { y registro de tierras } \\
\text { rurales en el Perú, } \\
\text { tercera etapa (PTRT3) }\end{array}$ & $\begin{array}{l}\text { UEGPS- } \\
\text { MINAGRI }\end{array}$ & 0 & 403 & 3.000 .000 & 0 & 2020 & $\begin{array}{l}\text { Amazonas, } \\
\text { Cajamarca, San } \\
\text { Martín, Junín, } \\
\text { Huánuco, } \\
\text { Ucayali, Cusco, } \\
\text { Apurímac, } \\
\text { Puno, Loreto }\end{array}$ \\
\hline $\begin{array}{l}\text { 8. FIP (Programa de } \\
\text { Inversión Forestal) } \\
\text { Tarapoto-Yurimaguas } \\
\text { en los departamentos } \\
\text { de San Martín y Loreto }\end{array}$ & PNCB & 2 & 5 & 70.000 & 25 & 2020 & $\begin{array}{l}\text { San Martín y } \\
\text { Loreto }\end{array}$ \\
\hline $\begin{array}{l}\text { 9. FIP Corredor Puerto } \\
\text { Maldonado - Iñapari } \\
\text { y la Reserva Comunal } \\
\text { Amarakaeri, en el } \\
\text { departamento de } \\
\text { Madre de Dios }\end{array}$ & PNCB & 0 & 8 & 45.000 & 17 & 2020 & Madre de Dios \\
\hline $\begin{array}{l}\text { 10. FIP Atalaya, en } \\
\text { el departamento de } \\
\text { Ucayali }\end{array}$ & PNCB & 2 & 4 & & 20 & 2020 & Ucayali \\
\hline
\end{tabular}

Fuente: MINAM-DCI (2018) (presentación realizada durante Mesa Entregable 4 - DCI).

*Acrónimos utilizados en esta tabla:

DEVIDA - Comisión Nacional para el Desarrollo y Vida sin Drogas

PNCB - Programa Nacional de Conservación de Bosques

FIP - Programa de Inversión Forestal (por sus siglas en inglés)

PTRT3 - Proyecto Catastro, Titulación y Registro de Tierras Rurales en el Perú,

Tercera Etapa
PNUD - Programa de Naciones Unidas para el Desarrollo CONAP - Confederación de Nacionalidades Amazónicas del Perú AIDESEP - Asociación Interétnica de Desarrollo de la Selva Peruana UEGPS - Unidad Ejecutora Gestión de Proyectos Sociales, del Proyecto PTRT3 


\section{Referencias}

[AIDESEP] Asociación Interétnica de Desarrollo de la Selva Peruana. 2016. Las demandas territoriales de los pueblos indígenas amazónicos del Perú. Lima: AIDESEP.

[AIDESEP] Asociación Interétnica de Desarrollo de la Selva Peruana. 2013. Territorialidad y titularidad en la Amazonía Norte del Perú, Alto Amazonas y Datem del Marañón - Pueblos Achuar, Kukama, Shapra, Kandozi, Shiwilo. Lima: AIDESEP/CORPI. Baldovino S. 2016. Una primera mirada: Situación legal de la tenencia de tierras rurales en Perú. Lima: SPDA.

Blackman A, Corral L, Lima ES, Asner G. 2017. Titling indigenous communities protects forests in the Peruvian Amazon. Proc Natl Acad Sci USA 114:4123-4128.

Bolaños, O. 2017. Resumen Político: Los derechos de las mujeres indígenas y los desafíos para los proyectos de titulación de la propiedad comunal en el Perú. Lima: RRI, ONAMIAP, CIFOR.

[CIFOR] Center for International Forestry Research y [WRI] World Resources Institute. 2017. Report analyzing formalization of native communities and company procedures in Peru. Technical report. Lima: CIFOR.

Defensoría del Pueblo. 2014. Análisis de la política pública sobre reconocimiento y titulación de las comunidades campesinas y nativas. Informe N. ${ }^{\circ}$ 002-2014-DP/AMASPPI-PPI. Lima: Defensoría del Pueblo.

Durán R, Monterroso I y Larson AM. 2018. Género e interculturalidad en la formalización de las comunidades nativas en Perú: Desafíos y recomendaciones. InfoBrief. Bogor, Indonesia: CIFOR. En edición.

[IBC] Instituto del Bien Común. 2016. Tierras comunales: Más que preservar el pasado es asegurar el futuro. El estado de las comunidades indígenas en el Perú. Informe 2016. Lima: Instituto del Bien Común.

[MINAM] Ministerio del Ambiente. 2016. La conservación de bosques en Perú (2011-2016): Conservando los bosques en un contexto de cambio climático como aporte al crecimiento verde. Consultado el 19 de febrero de 2016. http://www. minam.gob.pe/informessectoriales/wp-content/uploads/ sites/112/2016/02/11-La-conservaci\%C3\%B3n-de-bosques-en-elPer\%C3\%BA.pdf

[MINAM-DCl] Ministerio del Ambiente - Declaración Conjunta de Intenciones. 2018. Presentación Mesa Entregable 4 - Declaración Conjunta de Intenciones. Presentación realizada durante Primera ronda: Mesas Técnicas, 13 de febrero de 2018.

Monterroso I, Cronkleton P, Pinedo D y Larson AM. 2017. Reclaiming collective rights: Land and forest tenure reforms in Peru (1960-2016). Working Paper 224. Bogor, Indonesia: Center for International Forestry Research (CIFOR). Traducción al español: Working Paper 227].

Monterroso I y Larson AM. 2018. Desafíos del proceso de formalización de derechos de CC. NN. en Perú. InfoBrief. Bogor, Indonesia: CIFOR. En edición.

Red Muqui y GRUFIDES. 2015. Paquetes normativos 2013-2015 y su impacto en los derechos de las personas en el Perú: Análisis. Fecha de consulta: 9 de diciembre de 2016. http://www. grufides.org/sites/default/files//documentos/documentos/ Paquetazos\%20Antiambientales\%20Doc.\%20An\%C3\%A1 lisis\%20 Final.pdf

Schleicher J, Peres C A, Amano T, Llactayo W, y Leader-Williams N. 2017. Conservation performance of different conservation governance regimes in the Peruvian Amazon. Sci. Rep. 7, 11318.

[SICNA-IBC] Sistema de Información sobre Comunidades Nativas de la Amazonía Peruana - Instituto del Bien Común. 2016. Sistema de Información sobre Comunidades Nativas de la Amazonía Peruana. Perú: IBC. http://www.ibcperu.org/ servicios/sicna/

Zamora A y Monterroso I. 2017. Visiones regionales y locales sobre la inseguridad de tenencia en Loreto y Madre de Dios. InfoBrief 198. Bogor, Indonesia: Center for International Forestry Research (CIFOR)

\begin{tabular}{|c|c|c|}
\hline CGIAR & $\begin{array}{l}\text { PROGRAMA DE } \\
\text { INVESTIGACIÓN SOBRE } \\
\text { Bosques, Árboles y } \\
\text { Agroforestería }\end{array}$ & $\begin{array}{l}\text { El Programa de Investigación del CGIAR sobre Bosques, Árboles y Agroforestería (FTA) es el } \\
\text { programa de investigación para el desarrollo más grande del mundo, dedicado a mejorar el papel } \\
\text { de bosques, árboles y la agroforestería para el desarrollo sostenible, seguridad alimentaria, y frente } \\
\text { al cambio climático. CIFOR dirige el programa FTA en asociación con Bioversity International, CATIE, } \\
\text { CIRAD, ICRAF, INBAR y TBI. }\end{array}$ \\
\hline
\end{tabular}
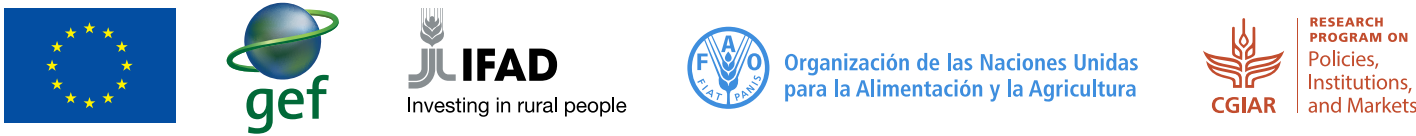
desarrollando las capacidades de sus socios y dialogando activamente con todos los actores involucrados, para informar sobre las políticas y las prácticas que afectan a los bosques y a las personas. CIFOR es un centro de investigación CGIAR y lidera su Programa de Investigación sobre Bosques, Árboles y Agroforestería (FTA por sus siglas en inglés). Nuestra sede central se encuentra en Bogor, Indonesia, y contamos con oficinas en Nairobi, Kenia; Yaundé, Camerún; y Lima, Perú. 